\title{
Pedicled Supraclavicular Artery Island Flap Versus Free Radial Forearm Flap: Perioperative Outcomes in Head and Neck Reconstruction
}

Jose A. González-García ${ }^{1}$, Carlos M. Chiesa Estomba Sr. ${ }^{1}$, Jon A. Sistiaga-Suarez ${ }^{1}$, Ekhiñe Larruscain ${ }^{2}$, Juan D. Urazan-Murcia ${ }^{1}$, Xabier Altuna ${ }^{1}$

1. ENT - Head and Neck Surgery, Hospital Universitario Donostia, San Sebastian, ESP 2. ENT - Head and Neck Surgery, Hospital Universitario Donostia, Donostia, ESP

Corresponding author: Carlos M. Chiesa Estomba Sr., chiesaestomba86@gmail.com

\section{Abstract}

Introduction: Radial forearm free flap (RFFF) and supraclavicular artery island flap (SCAIF) are some of the most common fasciocutaneous flaps used for head and neck $(\mathrm{H} \& \mathrm{~N})$ reconstruction.

Material and methods: A retrospective analysis of clinical data and outcomes of 31 consecutive patients who underwent H\&N reconstruction using either SCAIF or RFFF over a three-year period, aiming to compare the surgical outcomes of the SCAIF and the RFFF in $\mathrm{H} \& \mathrm{~N}$ reconstruction.

Results: Thirty-two flaps were performed in 31 patients (17 SCAIFs and 15 RFFFs). There was no difference in patient demographics between both groups. Hospital stay was longer in the SCAIF group (30.7 \pm 18.2 days (min: 9/max: 60) versus $19.2 \pm 15.8$ days (min: $7 / \max : 72$ ). Patients who underwent reconstruction with a SCAIF had shorter reconstructive procedure time; $74.4 \mathrm{~min}(\min : 60 / \mathrm{max}: 93)$ versus $147.8 \mathrm{~min}$ (min: 140/max: 187). Overall morbidity was not significantly different (SCAIF 52.7\% vs RFFF 39.9\%, p = NS). Global flap survival was higher without statistical significance in the RFFF group (100\%) versus the SCAIF group (70.7\%).

Conclusion: Despite the advantages related to the use of SCAIF like regarding the time spent in the reconstructive procedure. In our experience, the RFFF continues to be the most successful technique with similar perioperative outcomes and fewer complication rates. In this vein, both techniques can be reasonably used to reconstruct post-ablative H\&N defects. However, in our experience, the use of SCAIF may lengthen hospital length of stay probably due to the augmented risk of flap failure.

Review began 01/30/2021 Review ended 02/04/2021 Published 02/08/2021

\section{() Copyright 2021} González-García et al. This is an open access article distributed under the terms of the Creative Commons Attribution License CC-BY 4.0., which permits unrestricted use, distribution, and reproduction in any medium, provided the original author and source are credited.
Categories: Otolaryngology, Plastic Surgery

Keywords: free tissue flap, pedicled flap, head, neck, reconstruction

\section{Introduction}

Head and neck $(H \& N)$ reconstructive surgery aims to solve those problems occurring post tumor ablative resection from an anatomical region with multiple implications over the patient's quality of life. Essential functions like speech, mastication, swallowing, or breathing can be affected due to these ablative procedures. Also, the aesthetic defects related to the surgery can be sometimes unacceptable. In this vein, the ideal flap for $\mathrm{H} \& \mathrm{~N}$ reconstruction procedures should appropriately restore both form and function of the defect and in just one surgical act.

Free flaps are considered as the first option for complex or tridimensional soft tissue reconstruction in the $H \& N$ area, mainly the radial forearm free flap (RFFF) and the anterolateral thigh flap, both considered the workhorse flaps by $\mathrm{H} \& \mathrm{~N}$ reconstructive surgeons [1-5]. However, some patients may not be suitable for free tissue transfer (FTT) because of older age, poor nutritional status, medical comorbidities, vascular problems often associated with high tobacco or alcohol consumption, the higher risk of prolonged surgeries [6], or the lack of recipient's vessels because of previous treatments, especially in patients with metachronous or recurrent tumors [7]. Moreover, FTT requires considerable technical expertise, a longer learning curve, special instrumentation, specialized post-operative monitoring and care, and prolonged hospital stay.

In the last years, there is a resurgence of interest in the use of pedicled flaps in $\mathrm{H} \& \mathrm{~N}$. The pectoralis major flap and previously the deltopectoral flap were considered the workhorse flap in $\mathrm{H} \& \mathrm{~N}$ reconstruction historically. However, its thickness, limited length, and bulky pedicle [8] were considered the main drawback of this flap that supports the increasing use of the supraclavicular artery island flap (SCAIF) as a proper fasciocutaneous pedicled flap. The SCAIF represents an easy to harvest flap composed of a thin and pliable skin paddle. Due to flap versatility, it is useful for soft tissue defects in the facial, temporal, or parotid region, and upper aerodigestive reconstruction including the oral cavity, pharynx, or larynx [9-15]. The SCAIF elevation leaves negligible morbidity in the donor site with minimal postoperative care needs, making 
Literature comparing both techniques is scarce. However, some aspects have been considered previously, like short-term postoperative outcomes demonstrating that both options are reasonable for defects that can be reached by the SCAIF pedicle, with a little advantage in favor of the SCAIF [18-21].

This work aims to evaluate surgical outcomes between two groups of consecutive patients needing reconstruction with either a SCAIF or an RFFF in terms of clinical and demographic data, length of stay, operative time, and flap-related complications.

\section{Materials And Methods}

After the approval of the Ethics Committee of Donostia University Hospital, thirty-one patients (21 men and 10 women) who suffered from a primary $\mathrm{H} \& \mathrm{~N}$ cancer, and underwent soft tissue reconstruction with either SCAIF or RFFF between January 2017 and January 2020, were evaluated retrospectively. All patients were evaluated in the $\mathrm{H} \& \mathrm{~N}$ cancer multidisciplinary committee, and reconstruction was chosen depending on tumor and patient characteristics. All flaps were elevated by the first two authors. Both reconstructive surgical techniques were performed following the standard techniques. Patients younger than 18 years, those who need a composite reconstruction, or those when other types of flaps were used were not included.

Demographic and clinical data were collected and compared between both groups. Age, sex, comorbidities, alcohol and tobacco consumption, hospital stay, flap size, reconstructive surgical time, type of surgery, TNM stage (seventh edition), histology, and tumor differentiation data were extracted from the medical records. Also, specific flap-related complications were investigated. Total reconstructive procedure time includes flap elevation, insetting, and in the case of an RFFF, the time spent in the microsurgical venous and arterial anastomosis. In RFFF reconstructions, only one vein anastomosis, including the superficial and deep venous systems was performed. In five cases, the SCAIF was used as a back-up flap, while just in one case the RFFF was used as a back-up flap. In our institution, all FTT patients spend 24 hours in an intermediate care unit.

Descriptive results were expressed as mean \pm standard deviation. The Shapiro-Wilk test was used to assess the normal distribution between both groups. For dichotomous variables, Fisher's exact test and Pearson's Chi-square test were used. A p-value $<0.05$ was considered significant. Because the main target of this study was to analyze both reconstructive techniques, Student's t-test was used for quantitative variables. The confidence interval (95\%) was calculated. Regarding surgical time, the authors just compare the time spent during the reconstructive procedure and not the total surgical time.

\section{Results}

Patient's demographic data (medical background, alcohol and tobacco consumption, hospital length of stay, mean reconstructive surgical time, type of surgery, TNM stage, and histologic findings) are presented in Table 1.

\begin{tabular}{|c|c|c|c|c|c|}
\hline Variable & SCAIF & $\%$ & RFFF & $\%$ & p-Value \\
\hline \multicolumn{6}{|l|}{ Sex } \\
\hline Male & 11 & 68.8 & 10 & 66.7 & 0.001 \\
\hline Female & 5 & 31.3 & 5 & 33.3 & \\
\hline Flaps & 17 & 100 & 15 & 100 & \\
\hline Main flap & 12 & 70.5 & 14 & 93.3 & \\
\hline Back-up flap & 5 & 29.5 & 1 & 6.7 & \\
\hline Mean age & \multicolumn{2}{|c|}{$71 \pm 12$ years (min: 50/max: 91$)$} & \multicolumn{2}{|c|}{$56 \pm 10$ years (min: $49 / \max : 72)$} & 0.133 \\
\hline \multicolumn{6}{|l|}{ Comorbidities } \\
\hline DM & 6 & 37.5 & 1 & 6.7 & 0.125 \\
\hline HTA & 8 & 50 & 5 & 33.3 & 0.129 \\
\hline $\mathrm{BMI}<18.5$ & 3 & 18.8 & 1 & 6.7 & 0.101 \\
\hline Obesity & 5 & 31.3 & 3 & 20 & 0.12 \\
\hline COPD & 3 & 18.8 & 2 & 13.3 & 0.101 \\
\hline
\end{tabular}




\section{Cureus}

\begin{tabular}{|c|c|c|c|c|c|}
\hline Heart disease & 8 & 50 & 2 & 13.3 & 0.129 \\
\hline \multicolumn{5}{|l|}{ Smoker } & \multirow{4}{*}{0.209} \\
\hline$<20$ packs per year & 4 & 25 & 2 & 13.3 & \\
\hline$>20$ packs per year & 7 & 43.8 & 9 & 60 & \\
\hline No & 5 & 31.3 & 4 & 26.7 & \\
\hline \multicolumn{5}{|l|}{ Alcohol intake } & \multirow{3}{*}{0.128} \\
\hline$<70 \mathrm{~g}$ per day & 10 & 62.5 & 7 & 46.7 & \\
\hline$>70$ g per day & 6 & 37.5 & 8 & 53.3 & \\
\hline Mean hospital stay & \multicolumn{2}{|c|}{$31 \pm 18$ days (min: $9 / \max : 60)$} & \multicolumn{2}{|c|}{$19 \pm 16$ days (min: $7 / \max : 72)$} & 0.001 \\
\hline \multicolumn{5}{|l|}{ Type of surgery } & \multirow{8}{*}{0.062} \\
\hline Total laryngectomy & 3 & 18.8 & 3 & 20 & \\
\hline Oropharyngectomy & 1 & 6.3 & 2 & 13.3 & \\
\hline Salivary gland tumor (parotid) & 1 & 6.3 & 0 & 0 & \\
\hline Oral cavity & 4 & 25 & 9 & 60 & \\
\hline Skin cancer temporal bone and zygoma & 2 & 12.5 & 0 & 0 & \\
\hline Cutaneous defect & 5 & 31.3 & 0 & 0 & \\
\hline Skin cancer-midfacial resection & 0 & 0 & 1 & 6.7 & \\
\hline \multicolumn{5}{|l|}{ T stage } & \multirow{4}{*}{0.705} \\
\hline T2 & 2 & 12.5 & 3 & 20 & \\
\hline T3 & 2 & 12.5 & 3 & 20 & \\
\hline T4a & 12 & 75 & 9 & 60 & \\
\hline \multicolumn{5}{|l|}{$\mathrm{N}$ stage } & \multirow{8}{*}{0.724} \\
\hline No & 9 & 56.3 & 8 & 53.3 & \\
\hline N1 & 3 & 18.8 & 1 & 6.7 & \\
\hline $\mathrm{N} 2 \mathrm{a}$ & 0 & 0 & 0 & 0 & \\
\hline $\mathrm{N} 2 \mathrm{~b}$ & 2 & 12.5 & 0 & 0 & \\
\hline $\mathrm{N} 2 \mathrm{c}$ & 0 & 0 & 3 & 20 & \\
\hline N3a & 1 & 6.3 & 0 & 0 & \\
\hline N3b & 0 & 0 & 3 & 20 & \\
\hline \multicolumn{5}{|l|}{ M stage } & \multirow{2}{*}{1} \\
\hline M0 & 16 & 100 & 15 & 100 & \\
\hline \multicolumn{5}{|l|}{ Histology } & \multirow{3}{*}{1} \\
\hline $\operatorname{scc}$ & 15 & 93.8 & 15 & 100 & \\
\hline ACC & 1 & 6.3 & & & \\
\hline \multicolumn{5}{|l|}{ Differentiation grade (SCC) } & \multirow{4}{*}{0.162} \\
\hline Well & 4 & 25 & 1 & 6.7 & \\
\hline Moderately & 8 & 80 & 11 & 73.3 & \\
\hline Poorly & 4 & 25 & 3 & 20 & \\
\hline
\end{tabular}

TABLE 1: Demographic and clinical data analysis. 


\section{Cureus}

SCAIF: supraclavicular artery island flap, RFFF: Radial forearm free flap, DM: diabetes mellitus, AHT: arterial hypertension, COPD: chronic obstructive pulmonary disease, SCC: squamous cell carcinoma, ACC: adenoid cystic carcinoma.

The distribution between both groups was normal according to the Shapiro-Wilk test $(\mathrm{p}=0.130)$. Thirty-two flaps were elevated in 31 patients (one patient needed two SCAIF). The SCAIF was used in 16 patients (11 men and five women), and the RFFF was used in 15 patients (10 men and five women). The need for fasciocutaneous flaps was more frequent in men (Fisher - $\mathrm{p}=0.001$ ). We also tend to use the SCAIF in older patients, $70.6 \pm 11.8$ years ( $\min : 50$ /max: 91 ), while the RFFF is used in younger patients, $56.1 \pm 10.3$ years (min: 49/max: 72; Student T-test - $\mathrm{p}=0.130$ ). The overall mean area for the SCAIF donor site was 61.72 $\mathrm{cm}^{2}$ (min: $\left.36 \mathrm{~cm}^{2} / \max : 64 \mathrm{~cm}^{2}\right)$, compared with $78.2 \mathrm{~cm}^{2}\left(\min : 36 \mathrm{~cm}^{2} / \max : 98 \mathrm{~cm}^{2}\right)$ for the RFFF group $(\mathrm{p}=$ 0.056). The donor site defect was not quantified.

\begin{tabular}{|c|c|c|c|c|c|}
\hline Flap-related complications & SCAIF & $\%$ & RFFF & $\%$ & p-Value \\
\hline No & 8 & 47 & 9 & 60 & \multirow{6}{*}{0.502} \\
\hline Recipient site dehiscence & 1 & 5.8 & 2 & 13.3 & \\
\hline Donor site dehiscence & 3 & 17.6 & 4 & 26.6 & \\
\hline Partial flap necrosis & 3 & 17.6 & 0 & 0 & \\
\hline Total flap necrosis & 2 & 11.7 & 0 & 0 & \\
\hline Total & 17 & 10 & 15 & 100 & \\
\hline
\end{tabular}

\section{TABLE 2: Flap-related complications.}

SCAIF: supraclavicular artery island flap, RFFF: radial forearm free flap.

We found a significant difference between both groups according to the mean length of hospital stay; the RFFF group was discharged in $19 \pm 16$ days (min: 7/max: 72) while the SCAIF group was discharged in $31 \pm$ 18 days (min: 9/max: 60; $p=0.001$; Table 2). Regarding surgical time, it was significantly lower in the SCAIF group (74.4 min = min: 60/max: 93) compared to the RFF group (148 min = Min: 140/Max: 187; p=0.001). While the meantime to elevate an RFFF was $76 \mathrm{~min}(\min : 61 / \max$ : 95) and the meantime to elevate the SCAIF was $45 \min$ (min: 31/max: 66), being those differences statistically significant ( $\mathrm{p}=0.001$; Table 3 ).

\begin{tabular}{|c|c|c|c|c|}
\hline Variables & RFFF & SCAIF & p-Value & Cl \\
\hline Elevation & 76 min (min: 61/max: 95) & $45 \min (\min : 31 / \max : 66)$ & 0.001 & $30.9(95 \%=23.3$ to 38.7$)$ \\
\hline Insetting & 28 min (mın: 19/max: 37) & 30 mın (mın: 25/max: 41) & 0.397 & $(-) 1.63(95 \%=-5.51$ to 2.25$)$ \\
\hline Flap overall mean area & $78.2 \mathrm{~cm}^{2}\left(\min : 36 \mathrm{~cm}^{2} / \max : 98 \mathrm{~cm}^{2}\right)$ & $61.72 \mathrm{~cm}^{2}\left(\min : 36 \mathrm{~cm}^{2} / \max : 64 \mathrm{~cm}^{2}\right)$ & 0.056 & N/A \\
\hline Arterial anastomosis & 23.9 min (min: $21 / \max : 36)$ & N/A & & \\
\hline Venous anastomosis & 28.6 min (min: $25 / \max : 37)$ & N/A & & \\
\hline Total & $148 \min (\min : 140 / \max : 187)$ & 74 min (min: 60/max: 93) & 0.001 & $83.85(95 \%=74.5$ to 93.19$)$ \\
\hline
\end{tabular}

TABLE 3: Comparison of time specifically spent in the reconstructive procedure in each group.

SCAIF: supraclavicular artery island flap, RFFF: radial forearm free flap, Cl: confidence interval.

\section{Discussion}

Head and neck cancer surgery represents a mutilating procedure requiring, in some cases, the use of reconstructive procedures to assure aesthetic and functional outcomes. Although the use of FTT is still considered the workhorse in $\mathrm{H} \& \mathrm{~N}$ reconstruction, pedicled flaps may be regarded as an excellent option when FTT is not indicated. 
According to our findings, we demonstrated similar results between the use of both flaps in $\mathrm{H} \& \mathrm{~N}$ reconstruction, with the exception that those patients reconstructed with an RFFF were discharged earlier from the hospital, and the reconstructive procedure was shorter in the SCAIF group. Moreover, the elevation time does not affect total procedure time (resection + reconstruction) when, as in our case, the surgery is performed in a two-team approach. However, some authors advocate that when a SCAIF is used for H\&N reconstruction, flap elevation is challenging during the surgical resection even working as a two-team approach. Although when the contralateral neck is being dissected, the elevation of a SCAIF is still feasible, improving the total surgical time [18].

For experienced reconstructive surgeons, surgical time does not differ when performing an RFFF or a SCAIF [19], and the time needed to achieve an optimal result can be reduced with a growing surgeon's experience. As Sukato et al. demonstrated in a recently published systematic review of four studies comparing the use of the RFFF versus SCAIF in H\&N reconstruction [22]. In this review, surgical times for SCAIF and FTT cohorts were 368.68 (105.4) minutes and 462.1 (113.9) minutes, respectively, with a large effect size in favor of SCAIF [22].

When comparing hospital length, previous authors have reported no differences between SCAIF and RFFF groups [19] or reduced hospital stays in the SCAIF group [18]. However, in our experience, patients reconstructed with an RFFF are discharged earlier from the hospital. Being necessary to highlight that the nature of the hospital stays in both cases is different, because as we mention above, in our institution, those patients who undergo an FTT spend 24 hours in the ICU.

About those factors that can be related to the shorter length of stay, the absence of vascular complications in patients reconstructed with an RFFF in our study can be one of the main reasons. By contrast, five patients needed additional surgeries in the SCAIF group due to partial or total flap necrosis, consequently incrementing the total length of hospital stay. Remarkably, our first five cases of H\&N reconstruction with a SCAIF were all successful [17] and without complications. Nevertheless, such good results might be also due to the small study sample and complications can be expected when incrementing the number of patients. These complications could be related to older age or comorbidities, as this group tends to include patients not suitable for FTT, with a negative selection bias. As prior authors have been remarked, the SCAIF improved surgical efficiency, because just one surgeon can be enough to elevate the flap, minimizing the use of operating room personnel requirements [19].

Regarding surgical complications, we could not find any statistical difference among both groups, despite the absence of complications in those patients treated with an RFFF, compared to the total loss of two flaps and the development of three partial flap necrosis in the SCAIF group. Also, Granzow et al. [18], Kozin et al. [19], and Welz et al. [20] did not find statistical differences regarding complications. However, Zhang et al. [21] reported a significant increase in donor-site complications in the RFFF group, mainly hypertrophic scarring and partial skin graft necrosis without differences in recipient-site complications. When systematically compared, it seems that SCAIF prevents total flap loss while RFFF prevents partial flap loss without statistical significance. Also, RFFF use appears to increase donor-site dehiscence, and SCAIF seems to increase recipient-site dehiscence, although differences could have been missed because of short samples reducing statistical power [22].

The addition of pedicled SCAIFs for $\mathrm{H} \& \mathrm{~N}$ reconstruction can significantly reduce operative times and implies less surveillance of flap vascular status in the first days after surgery, with the extra benefit of reduced postoperative intensive care length of stay as described by the previous authors [18]. Another advantage of using the SCAIF flap as a fasciocutaneous alternative to the RFFF in patients not suitable for FTT or by-election of the surgical team is that hospital costs can be reduced as FTT increases costs when compared in global or by type of oncologic resection [19,20]. Regarding functional outcomes, Zhang et al. [21] reported similar outcomes in the quality of speech and swallowing, when performing hemyglossectomy reconstruction. Still, functional results in different H\&N subsites and quality of life after surgery protocols should be obtained in the future to disclose more accurate results.

The main limitations of our study, as in previous similar reports, can be related to the small sample size, making a challenge to establish proper comparison among our results (hospital stay, complication rates, quality of life, etc.), the difference according to each anatomical subsite treated (larynx, oropharynx, salivary glands, etc.), the risk of selection bias, the lack of cost analysis and the lack of a proper functional outcome evaluation. Moreover, the fact that measures of wound healing are inherently subjective and may vary according to each surgeon makes it challenging to establish a rigorous comparison. In this way, we decide to continue with our prospective data collection to re-evaluate our results in the future.

\section{Conclusions}

Despite those well-known advantages related to the use of the SCAIF regarding the time spent during the reconstructive procedure or the cost-effectiveness, in our experience, the RFFF continues to be the most successful technique with similar perioperative outcomes and fewer complication rates. In this vein, both techniques can be reasonably used to reconstruct post ablative $H \& N$ defects. However, in our experience, the use of SCAIF may enlarge hospital length of stay probably due to augmented risk of flap failure. 


\section{Additional Information \\ Disclosures}

Human subjects: Consent was obtained or waived by all participants in this study. HUD issued approval $\mathrm{CCH}$ - 172003. This retrospective study was approved by the IRB of Hospital Universitario Donostia in March 2020. Animal subjects: All authors have confirmed that this study did not involve animal subjects or tissue. Conflicts of interest: In compliance with the ICMJE uniform disclosure form, all authors declare the following: Payment/services info: All authors have declared that no financial support was received from any organization for the submitted work. Financial relationships: All authors have declared that they have no financial relationships at present or within the previous three years with any organizations that might have an interest in the submitted work. Other relationships: All authors have declared that there are no other relationships or activities that could appear to have influenced the submitted work.

\section{References}

1. Koshima I, Fukuda H, Utunomiya R, Soeda S: The anterolateral thigh flap; variations in its vascular pedicle . Br J Plast Surg. 1989, 42:260-2. 10.1016/0007-1226(89)90142-2

2. Soutar DS, McGregor IA: The radial forearm flap in intraoral reconstruction: the experience of 60 consecutive cases. Plast Reconstr Surg. 1986, 78:1-8. 10.1097/00006534-198607000-00001

3. Mühlbauer W, Herndl E, Stock W: The forearm flap. Plast Reconstr Surg. 1982, 70:336-44. 10.1097/00006534-198209000-00007

4. Yang GF, Chen PJ, Gao YZ, Liu XY, Li J, Jiang SX, He SP: Forearm free skin flap transplantation: a report of 56 cases. Br J Plast Surg. 1981, 50:162-5. 10.1016/s0007-1226(97)91363-1

5. Ferrandis Perepérez E, Antón Almero M, Guillén Martínez A, Martínez Hervás R, Pla Mocholí Á: Versatility of the radial forearm free flap in head and neck reconstruction. A study of 58 cases. Acta Otorrinolaringol Esp. 2020, 71:275-80. 10.1016/j.otorri.2019.10.001

6. Brady JS, Desai SV, Crippen MM, et al.: Association of anesthesia duration with complications after microvascular reconstruction of the head and neck. JAMA Facial Plast Surg. 2018, 20:188-95. 10.1001/jamafacial.2017.1607

7. Coca-Pelaz A, Rodrigo JP, Suárez C, et al.: The risk of second primary tumors in head and neck cancer: a systematic review. Head Neck. 2020, 42:456-66. 10.1002/hed.26016

8. Kruse AL, Luebbers HT, Obwegeser JA, Bredell M, Grätz KW: Evaluation of the pectoralis major flap for reconstructive head and neck surgery. Head Neck Oncol. 2011, 3:12. 10.1186/1758-3284-3-12

9. Epps MT, Cannon CL, Wright MJ, Chaffin AE, Newsome RE, Friedlander PL, Chiu ES: Aesthetic restoration of parotidectomy contour deformity using the supraclavicular artery island flap. Plast Reconstr Surg. 2011, 127:1925-31. 10.1097/PRS.0b013e31820cf40a

10. Levy JM, Eko FN, Hilaire HS, Friedlander PL, Melgar MA, Chiu ES: Posterolateral skull base reconstruction using the supraclavicular artery island flap. J Craniofac Surg. 2011, 22:1751-4. 10.1097/SCS.0b013e31822e62e9

11. Nicoli F, Orfaniotis G, Gesakis K, et al.: Supraclavicular osteo-cutaneous free flap: clinical application and surgical details for the reconstruction of composite defects of the nose. Microsurgery. 2015, 35:328-32. 10.1002/micr.22375

12. Fang SL, Zhang DM, Chen WL, Wang YY, Fan S: Reconstruction of full-thickness cheek defects with a folded extended supraclavicular fasciocutaneous island flap following ablation of advanced oral cancer. J Cancer Res Ther. 2016, 12:888-91. 10.4103/0973-1482.177497

13. Emerick KS, Herr MA, Deschler DG: Supraclavicular flap reconstruction following total laryngectomy . Laryngoscope. 2014, 124:1777-82. 10.1002/lary.24530

14. Henderson MM, Chiu ES, Jaffer AS: A simple approach of tubularizing the supraclavicular flap for circumferential phar-yngoesophageal defects. Plast Reconstr Surg. 2010, 126:28-29. 10.1097/PRS.0b013e3181dab4a5

15. Liu PH, Chiu ES: Supraclavicular artery flap: a new option for pharyngeal reconstruction . Ann Plast Surg. 2009, 62:497-501. 10.1097/SAP.0b013e31818c9d7d

16. Kokot N, Mazhar K, Reder LS, Peng GL, Sinha UK: The supraclavicular artery island flap in head and neck reconstruction: applications and limitations. JAMA Otolaryngol Head Neck Surg. 2013, 139:1247-55. 10.1001/jamaoto.2013.5057

17. González-García JA, Chiesa-Estomba CM, Sistiaga JA, Larruscain E, Álvarez L, Altuna X: Utility and versatility of the supraclavicular artery island flap in head and neck reconstruction. Acta Otorrinolaringol Esp. 2018, 69:8-17. 10.1016/j.otorri.2017.03.004

18. Granzow JW, Suliman A, Roostaeian J, Perry A, Boyd JB: Supraclavicular artery island flap (SCAIF) vs free fasciocutaneous flaps for head and neck reconstruction. Otolaryngol Head Neck Surg. 2013, 148:941-8. 10.1177/0194599813476670

19. Kozin ED, Sethi RK, Herr M, et al.: Comparison of perioperative outcomes between the supraclavicular artery island flap and fasciocutaneous free flap. Otolaryngol Head Neck Surg. 2016, 154:66-72. 10.1177/0194599815607345

20. Welz C, Canis M, Schwenk-Zieger S, Spiegel JL, Weiss BG, Pilavakis Y: Oral cancer reconstruction using the supraclavicular artery island flap: comparison to free radial forearm flap. J Oral Maxillofac Surg. 2017, 75:2261-9. 10.1016/j.joms.2017.02.017

21. Zhang S, Chen W, Cao G, Dong Z: Pedicled supraclavicular artery island flap versus free radial forearm flap for tongue reconstruction following hemiglossectomy. J Craniofac Surg. 2015, 26:527-30. 10.1097/SCS.0000000000002031

22. Sukato DC, Timashpolsky A, Ferzli G, Rosenfeld RM, Gordin EA: Systematic review of supraclavicular artery island flap vs free flap in head and neck reconstruction. Otolaryngol Head Neck Surg. 2019, 160:215-22. $10.1177 / 0194599818803603$ 